This item was submitted to Loughborough's Research Repository by the author.

Items in Figshare are protected by copyright, with all rights reserved, unless otherwise indicated.

\title{
CVBEM solution for De Saint-Venant orthotropic beams under coupled bending and torsion
}

\section{PLEASE CITE THE PUBLISHED VERSION}

http://dx.doi.org/10.1007/s00707-014-1233-y

\section{PUBLISHER}

(c) Springer Vienna

\section{VERSION}

AM (Accepted Manuscript)

\section{PUBLISHER STATEMENT}

This work is made available according to the conditions of the Creative Commons Attribution-NonCommercialNoDerivatives 4.0 International (CC BY-NC-ND 4.0) licence. Full details of this licence are available at: https://creativecommons.org/licenses/by-nc-nd/4.0/

\section{LICENCE}

CC BY-NC-ND 4.0

\section{REPOSITORY RECORD}

Barone, Giorgio, Antonina Pirrotta, and Roberta Santoro. 2019. "CVBEM Solution for De Saint-venant Orthotropic Beams Under Coupled Bending and Torsion". figshare. https://hdl.handle.net/2134/16763. 


\title{
CVBEM solution for De Saint-Venant orthotropic beams under coupled bending and torsion.
}

Giorgio Barone $^{(1)}$, Antonina Pirrotta ${ }^{(2)}$, Roberta Santoro ${ }^{(3)} *$

${ }^{(1)}$ Facoltà di Ingegneria ed Architettura, Università degli studi di Enna "Kore”, Cittadella Universitaria, 94100 Enna, Italy

${ }^{(2)}$ Dipartimento di Ingegneria Civile, Ambientale, Aerospaziale, Dei Materiali (DICAM),

Università Degli Studi di Palermo, Viale delle Scienze, 90128 Palermo, Italy

${ }^{(3)}$ Dipartimento di Ingegneria Civile, Informatica, Edile, Ambientale e Matematica Applicata (DICIEAMA)

Università degli studi di Messina, C.da Di Dio, 98166 Messina, Italy

E-mail: ingbarone@gmail.com, antonina.pirrotta@unipa.it, roberta.santoro@unime.it

*Corresponding author: Tel:+390903977200, E-mail: roberta.santoro@unime.it

\begin{abstract}
The aim of this paper is to provide a solution for the coupled flexure-torsion De Saint Venant problem for orthotropic beams taking full advantage of the Complex Variable Boundary Element Method (CVBEM) properly extended by using a complex potential function whose real and imaginary parts are related to the shear stress components, the orthotropic ratio and the Poisson coefficients.

The proposed method returns the complete stress field and the unitary twist rotation of the crosssection at once by performing only line integrals.

Numerical applications have been reported to show the validity and the efficiency of the proposed modified CVBEM to handle shear stress problems in presence of orthotropic materials.
\end{abstract}

Keywords: De Saint-Venant beam; Flexure-torsion problem; orthotropic material; CVBEM; Complex potential function. 


\section{Introduction}

In recent years the advent of composite materials (obtained by the combination, on a macroscopic scale, of two or more basic materials) makes indispensable to account for the material orthotropy, which is due to the properties and orientation of the constituents and leads to mechanical properties quite different from those of isotropic materials.

In the theory of strength of materials, the evaluation of shear stress fields in De Saint-Venant beam due to external shear forces and twist moments has been widely investigated. Nevertheless, because of its inherent mathematical difficulties, analytical solutions are known only for a few simple cross-sectional shapes both in the isotropic and in the orthotropic case [1-3].

Results in terms of shear stresses distribution for orthotropic beams subjected to flexure-torsion actions are provided by Lekhnitskii [4] and Tolf [5] through series expansion for a limited class of cross-sections. In all other cases, numerical methods are used, the most commonly employed being Finite Element Method (FEM) and Boundary Element Method (BEM).

Within the framework of FEM, a finite element approach was employed in Kosmatka and Dong [6] to calculate displacement and stress distributions for an homogeneous prismatic beam of arbitrary section and rectilinear anisotropy under flexure-torsion action. A Ritz-based power series method as reported in Kosmatka [7] was used to analyze the behaviour of a general anisotropic material with arbitrary cross-section.

Both FEMs and Ritz power series methods require the whole cross-section to be discretized into area elements. Although solutions via FEM approach are of simple and immediate use in order to reach a good accuracy, in case of complicated cross-sections large number of elements are required, yielding to inconvenient meshing processes and high computational effort. Similarly the numerical integration in the Ritz based methods requires a large number of integration points because of the high order of the power series.

On the other hand, BEM requires instead only discretization of the boundary, resulting more convenient when dealing with complicated shapes and achieving high accuracy with a small number of boundary elements.

In flexure analysis, a BEM procedure with constant elements has been used for the calculation of the shear centre location in Chou [8]. 
A stress function solution and a displacement solution employing the BEM for the general transverse shear loading problem of homogeneous and composite prismatic beams of arbitrary cross-section was presented in Mokos an Sapountzakis [9,10] and Sapountzakis and Protonotariou [11], respectively. A boundary element model for the orthotropic analysis of De Saint Venant flexure-torsion problem was presented in Gaspari and Aristodemo [12] where the differential equations governing the shear stress field are converted in three Neumann problems which form the basis of the boundary integral formulation and a quadratic B-spline approximation represents the boundary variables.

A comparison between different methods, including a displacement approach, a mixed approach and a potential stress approach for the solution of the flexuretorsion problem has been discussed in Lacarbonara and Paolone [13].

Alternatively, the problem can be formulated in terms of complex potential functions. Pure torsion problems in De Saint-Venant beams have, in fact, being studied using potential functions in terms of warping function [14,2,15].

Application of complex analysis in the boundary element methods result in Complex Variable Boundary Element Method (CVBEM) [16-18], Complex Polynomial Method (CPM) [19,20] and recently in Line Element-less Method (LEM) [21-24].

Both CVBEM and CPM [25] provide solutions of two-dimensional potential problems by generalization of the Cauchy integral formula into a boundary integral equation method. In particular, CVBEM is essentially based on two main approximations: $i$ ) the boundary of the studied domain is approximate into a polygonal line by selecting $n$ arbitrary nodes on the boundary itself and $i i$ ) the approximate complex potential function defined on each segment of the polygonal contour is used to provide the problem solution.

The CPM is based on the expansion of the complex potential function in Taylor series, whose unknown coefficients are evaluated by means of collocation points on the boundary.

Both methods have been successfully applied to the case of pure torsion problems in De Saint-Venant isotropic beam. To the best of the author's knowledge, only one application of CVBEM for orthotropic beam is provided in [26] limited to the case of a twist moment excitation. 
LEM works in terms of a novel complex potential function related directly with the shear stresses, satisfying the field equations in the whole domain and expanded in double-ended Laurent series. The unknown series coefficients are evaluated by a weak-form procedure imposing that the square net flux of the shear stresses across the boundary of the cross-section is minimum under the static equivalence condition. LEM allows to evaluate the complete stress field distribution and unitary twist rotation by using only line integrals without requiring meshing process neither of the domain nor the boundary. The idea of introducing this novel potential function in terms of shear stresses made it possible to take profit of complex analysis in a straightforward extension to study coupled shear and torsion problems [22].

Moreover, the LEM accomplished with a suitable transformation of coordinates [2] was also successfully extended providing solutions for the pure torsion case [27] and simultaneous action of torsion and shear forces [28] for orthotropic beams having simply and multiply-connected cross-section.

The same complex potential function in terms of shear stresses has been extended to CVBEM by the first two authors in [29] to solve pure torsion problem and for studying the case of flexure and torsion [30] with the advantage to evaluate at the same time both the complete shear stress field and the value of the twist rotation of the cross-section per unit length, performing only line integrals. For isotropic beams with boundary singularity problems, the CVBEM has been applied for the solution of the flexure-torsion problem in Barone et al. [31] using a novel formulation based on the definition of the complex potential of a fictitious vector field, analogous to the velocity field of an irrotational, incompressible flow.

Aim of this paper is the extension of procedure proposed in Barone and Pirrotta [30] for solving flexure-torsion problems in orthotropic beams taking full advantage of the complex potential function introduced in Santoro [28] whose real and imaginary parts are related to the shear stress components, the orthotropic ratio and the Poisson coefficients.

Numerical results have been reported to show the accuracy and the efficiency of the proposed CVBEM to handle flexure-torsion problems in presence of orthotropic material. 


\section{Theoretical background}

For the sake of clarity, this section introduces some basic concepts about De Saint Venant theory for beams subjected to shear and torsion actions, as well as appropriate symbols.

By definition, an orthotropic material has two or three mutually orthogonal planes of elastic symmetry, where mechanical properties are independent of direction. These independent elastic constants in the orthotropic constitutive equations are the three Young's moduli, the three Poisson's ratios, and the three shear moduli, in the following indicated with $E_{x}, E_{y}, E_{z} v_{z x}, v_{z y}, v_{x y}$ and $G_{z x}, G_{z y}, G_{x y}$, respectively. It's worth noting that $v_{i j} / E_{i}=v_{j i} / E_{j}$ where, in general, $v_{i j} \neq v_{j i}$.

Following the De Saint-Venant theory, it is assumed $\sigma_{x}=\sigma_{y}=\tau_{x y}=0$. Therefore the constitutive equations read:

$$
\varepsilon_{x}=-\frac{\nu_{z x}}{E_{z}} \sigma_{z} ; \varepsilon_{y}=-\frac{v_{z y}}{E_{z}} \sigma_{z} ; \varepsilon_{z}=\frac{1}{E_{z}} \sigma_{z} ; \gamma_{x y}=0 ; \gamma_{z y}=\frac{\tau_{z y}}{G_{z y}} ; \gamma_{z x}=\frac{\tau_{z x}}{G_{z x}}
$$

As in the isotropic case, the equilibrium equations are:

$$
\begin{gathered}
\frac{\partial \tau_{z x}}{\partial z}=0 ; \frac{\partial \tau_{z y}}{\partial z}=0 ; \frac{\partial \tau_{z x}}{\partial x}+\frac{\partial \tau_{z y}}{\partial y}=-\frac{\partial \sigma_{z}}{\partial z} \\
(2 a, b, c)
\end{gathered}
$$

while the compatibility equations become:

$$
\begin{aligned}
& \frac{\partial^{2} \varepsilon_{x}}{\partial y^{2}}+\frac{\partial^{2} \varepsilon_{y}}{\partial x^{2}}=0 ; \frac{\partial^{2} \varepsilon_{x}}{\partial z^{2}}+\frac{\partial^{2} \varepsilon_{z}}{\partial x^{2}}=0 ; \frac{\partial^{2} \varepsilon_{y}}{\partial z^{2}}+\frac{\partial^{2} \varepsilon_{z}}{\partial y^{2}}=0 ; \frac{\partial^{2} \varepsilon_{z}}{\partial x \partial y}=0 \\
& \frac{\partial^{2} \varepsilon_{y}}{\partial x \partial z}=\frac{1}{2} \frac{\partial}{\partial y}\left(\frac{\partial \gamma_{z y}}{\partial x}-\frac{\partial \gamma_{z x}}{\partial y}\right) ; \frac{\partial^{2} \varepsilon_{x}}{\partial y \partial z}=\frac{1}{2} \frac{\partial}{\partial x}\left(\frac{\partial \gamma_{z x}}{\partial y}-\frac{\partial \gamma_{z y}}{\partial x}\right)
\end{aligned}
$$


Taking into account Eqs.(1) and (2), the compatibility equations reported in Eq.(3) can be rewritten in terms of stresses as:

$$
\begin{aligned}
& v_{z x} \frac{\partial^{2} \sigma_{z}}{\partial y^{2}}+v_{z y} \frac{\partial^{2} \sigma_{z}}{\partial x^{2}}=0 ; \frac{\partial^{2} \sigma_{z}}{\partial x^{2}}=0 ; \frac{\partial^{2} \sigma_{z}}{\partial y^{2}}=0 ; \frac{\partial^{2} \sigma_{z}}{\partial x \partial y}=0 ; \\
& -\frac{v_{z y}}{E_{z}} \frac{\partial^{2} \sigma_{z}}{\partial x \partial z}=\frac{1}{2}\left(\frac{1}{G_{z y}} \frac{\partial^{2} \tau_{z y}}{\partial x \partial y}-\frac{1}{G_{z x}} \frac{\partial^{2} \tau_{z x}}{\partial y^{2}}\right) ;-\frac{v_{z x}}{E_{z}} \frac{\partial^{2} \sigma_{z}}{\partial y \partial z}=\frac{1}{2}\left(\frac{1}{G_{z x}} \frac{\partial^{2} \tau_{z x}}{\partial x \partial y}-\frac{1}{G_{z y}} \frac{\partial^{2} \tau_{z y}}{\partial x^{2}}\right)
\end{aligned}
$$

Let us consider a beam with three mutually orthogonal planes of elastic symmetry, one of them coinciding with the cross-section plane. The beam has length $L$, arbitrary cross-section with area $A$ and contour $C$. The reference system illustrated in Fig. 1 has been considered, with origin in the centroid of the fixed end, $z$ axis parallel to the beam generators, and $x$ and $y$ axes directed along the principal inertia axes of the cross-section and oriented as the material directions of orthotropy. It is assumed that shear forces $T_{x}(L), T_{y}(L)$ and torsion moment $M_{z}(L)$ are applied at the end cross-section.

Indicating by $I_{x}$ and $I_{y}$ the moments of inertia of the cross-section with respect to the $x$ and $y$ axes, the normal stress $\sigma_{z}(z)$ induced by bending moments $M_{x}(z)$ and $M_{y}(z)$ is:

$$
\sigma_{z}(z)=M_{x}(z) y / I_{x}-M_{y}(z) x / I_{y}
$$

The shear stresses $\tau_{z x}(x, y)$ and $\tau_{z y}(x, y)$, collected in the vector $\boldsymbol{\tau}^{\mathrm{T}}=\left[\begin{array}{ll}\tau_{z x} & \tau_{z y}\end{array}\right]$, are solutions of both the third equilibrium equation (Eq.2c), rewritten as

$$
\operatorname{div} \boldsymbol{\tau}=-\frac{\partial \sigma_{z}}{\partial z} \text { in } A
$$


and the compatibility equations (Eqs.4e and 4f) that can be rewritten in the following form

$$
\begin{aligned}
& \frac{\partial^{2} \sigma_{z}}{\partial x \partial z}\left(\frac{1}{2 G_{y z}}-\frac{v_{z y}}{E_{z}}\right)=-\frac{1}{2}\left(\frac{1}{G_{y z}} \frac{\partial^{2} \tau_{z x}}{\partial x^{2}}+\frac{1}{G_{x z}} \frac{\partial^{2} \tau_{x z}}{\partial y^{2}}\right) \\
& \frac{\partial^{2} \sigma_{z}}{\partial y \partial z}\left(\frac{1}{2 G_{x z}}-\frac{v_{z x}}{E_{z}}\right)=-\frac{1}{2}\left(\frac{1}{G_{x z}} \frac{\partial^{2} \tau_{z y}}{\partial y^{2}}+\frac{1}{G_{y z}} \frac{\partial^{2} \tau_{y z}}{\partial x^{2}}\right)
\end{aligned}
$$

The free-traction boundary condition along the contour of the cross-section, expressed in terms of stress components, $\tau_{z x}$ and $\tau_{z y}$, and of the outward normal vector $\mathbf{n}^{\mathrm{T}}=\left[\begin{array}{ll}n_{x} & n_{y}\end{array}\right]$ to the boundary $\mathrm{C}$, is:

$$
\tau_{z x} n_{x}+\tau_{z y} n_{y}=\boldsymbol{\tau}^{\mathrm{T}} \mathbf{n}=0 \text { on } C
$$

Moreover, the static equivalence conditions have to be satisfied, namely

$$
\int_{A} \tau_{z x} d A=T_{x} \quad ; \quad \int_{A} \tau_{z y} d A=T_{y} \quad ; \quad \int_{A}\left(\tau_{z y} x-\tau_{z x} y\right) d A=M_{z}
$$

By following Sokolnikoff [2], a simplified formulation of the De Saint Venant flexure-torsion problem for orthotropic materials is provided by introducing the so-called orthotropic ratio $R$ defined as

$$
R=\sqrt{G_{z y} / G_{z x}}
$$

and the following independent variables 


$$
\xi=R x \quad ; \eta=y
$$

(11a,b)

The flexure-torsion problem can be solved in the transformed domain $\bar{A}$ with boundary $\bar{C}$ with respect to the new introduced variables $\xi$ and $\eta$ (see Fig.2) .

In the transformed domain the governing equations appear similar to the isotropic case, as detailed in the following.

Indeed, the third equilibrium equation (Eq.2c) becomes:

$$
R \frac{\partial \tau_{z x}(\xi, \eta)}{\partial \xi}+\frac{\partial \tau_{z y}(\xi, \eta)}{\partial \eta}=-\frac{T_{y}}{I_{x}} \eta-\frac{T_{x}}{I_{y}} \frac{\xi}{R} \text { in } \bar{A}
$$

and the compatibility equations in $(7 a, b)$ can be expressed as:

$$
\nabla_{2} \tau_{z x}(\xi, \eta)=\left(\frac{2 v_{z y} G_{z x}}{E_{z}}-\frac{1}{R^{2}}\right) R \frac{\partial^{2} \sigma_{z}}{\partial \xi \partial z} \quad ; \quad \nabla_{2} \tau_{z y}(\xi, \eta)=\left(\frac{2 v_{z x} G_{z x}}{E_{z}}-1\right) \frac{\partial^{2} \sigma_{z}}{\partial \eta \partial z}
$$

where $\nabla_{2}[\bullet]=\frac{\partial^{2}[\bullet]}{\partial x^{2}}+\frac{\partial^{2}[\bullet]}{\partial y^{2}}$.

Eqs.(13a,b) can be rewritten as:

$$
\nabla_{2} \tau_{z x}(\xi, \eta)=\frac{\left(m_{2}-1\right)}{R^{2}} \frac{T_{x}}{I_{y}} ; \nabla_{2} \tau_{z y}(\xi, \eta)=\left(m_{1}-1\right) \frac{T_{y}}{I_{x}}
$$

$(14 a, b)$

where the following positions have been made: 


$$
\frac{2 G_{z x} v_{z x}}{E_{z}}=m_{1} \quad ; \quad \frac{2 G_{z y} v_{z y}}{E_{z}}=m_{2}
$$

$(15 \mathrm{a}, \mathrm{b})$

The boundary condition expressed in Eq.8 turns, in the transformed domain, in:

$$
R \tau_{z x}(\xi, \eta) \bar{n}_{\xi}+\tau_{z y}(\xi, \eta) \bar{n}_{\eta}=\boldsymbol{\tau}^{\mathrm{T}} \overline{\mathbf{n}}=0 \quad \text { on } \quad \bar{C}
$$

with $\overline{\mathbf{n}}^{\mathrm{T}}=\left[\begin{array}{ll}\bar{n}_{\xi} & \bar{n}_{\eta}\end{array}\right]$.

Finally, the static equivalence conditions are changed into:

$$
\frac{1}{R} \int_{\bar{A}} \tau_{z x} d \bar{A}=T_{x} \quad ; \quad \frac{1}{R} \int_{\bar{A}} \tau_{z y} d \bar{A}=T_{y} \quad ; \quad \frac{1}{R} \int_{\bar{A}}\left(\tau_{z y} \frac{\xi}{R}-\tau_{z x} \eta\right) d \bar{A}=M_{z} .
$$

Once the set of equation fully characterizing the flexure-torsion problem on a generic orthotropic beam is defined, the achieved solution by using complex analysis is presented into the next section.

\section{Complex potential function formulation}

Formulation of the flexure-torsion problem for the orthotropic beam in the transformed domain $\bar{A}$ allows to take full advantage of the theory of analytic complex functions. The boundary value problem is formulated by introducing a complex potential function $F(\zeta)$, analytic in all the transformed domain. Real and imaginary parts of the complex potential, defined as $\chi_{x}(\xi, \eta)$ and $\chi_{y}(\xi, \eta)$, are related to the shear stress components, the orthotropic ratio and the Poisson coefficients, as recently proposed in Santoro [28]: 


$$
\chi_{x}(\xi, \eta)=\operatorname{Re}[F(\zeta)]=R \tau_{z x}(\xi, \eta)-\left(m_{2}-1\right)\left(-\frac{m_{1}}{\left(m_{2}-1\right)} \frac{T_{y}}{I_{x}} \xi \eta+\frac{T_{x}}{2 I_{y}} \frac{\xi^{2}}{R}\right)+R G_{z x} \bar{\theta} \eta
$$

$$
\chi_{y}(\xi, \eta)=\operatorname{Im}[F(\zeta)]=-\tau_{z y}(\xi, \eta)+\left(m_{1}-1\right)\left(-\frac{m_{2}}{\left(m_{1}-1\right)} \frac{T_{x}}{I_{y}} \frac{\xi \eta}{R}+\frac{T_{y}}{2 I_{x}} \eta^{2}\right)+R G_{z x} \bar{\theta} \xi
$$

where $\bar{\theta}$ is an unknown constant.

$\chi_{x}(\xi, \eta)$ and $\chi_{y}(\xi, \eta)$ are harmonic real functions:

$$
\nabla^{2} \chi_{x}(\xi, \eta)=0 ; \quad \nabla^{2} \chi_{y}(\xi, \eta)=0 \quad \text { in } \bar{A}
$$

and conjugate harmonic functions, that is, the Cauchy-Riemann conditions hold true:

$$
\frac{\partial \chi_{x}}{\partial \xi}=\frac{\partial \chi_{y}}{\partial \eta} ; \quad \frac{\partial \chi_{x}}{\partial \eta}=-\frac{\partial \chi_{y}}{\partial \xi} \quad \text { in } \bar{A}
$$

It is worth underline that Eqs.(19a,b) and (20a,b) constitute the conditions for $F(\zeta)$ being analytic and they revert to all governing equations valid in the

transformed domain $\bar{A}$, namely equilibrium and compatibility equations. This implies that the tangential stress due to shear induced torsion are taken into account, should the shear centre not coincide with the centroid of the crosssection.

\section{CVBEM for flexure-torsion problems in orthotropic media}

Let us consider again the beam as depicted in Fig. 2 with simply connected domain $\bar{A}$ bounded by $\bar{C}$ and subjected to external shear forces $T_{x}, T_{y}$ and torsion moment $M_{z}$. Following the formulation proposed by Whitley and Hromadka in [18], the potential function $F(\zeta)$ approximating the flexure-torsion boundary value problem is expressed as: 


$$
\begin{aligned}
& F(\zeta)=\alpha+\alpha_{0} \zeta+\sum_{k=1}^{n} \alpha_{k} f_{\zeta_{k}}(\zeta) \\
& f_{\zeta_{k}}(\zeta)=\left(\zeta-\zeta_{k}\right) \log _{\zeta_{k}}\left(\zeta-\zeta_{k}\right)
\end{aligned}
$$

where $n$ nodes $\zeta_{k}=\xi_{k}+i \eta_{k}$ have been selected on the contour $\bar{C}$ of the transformed domain and

$$
\begin{aligned}
& \alpha=a+i b \\
& \alpha_{0}=a_{0}+i b_{0} \quad(22 \mathrm{a}, \mathrm{b}, \mathrm{c}) \\
& \alpha_{k}=a_{k}+i b_{k}
\end{aligned}
$$

are complex unknown coefficients with $a, a_{0}, a_{k}, b, b_{0} b_{k} \in \mathbb{R}$.

Since the approximate complex potential function $F(\zeta)$ has to keep the requirements to be analytic in $\bar{A}$ and continuous in $\bar{A} \cup \bar{C}$ by using the series in Eqs.(21), it is necessary that the logarithms with respect to an opportune branch cut for each node $\zeta_{k}$ have to be precisely defined as extensively declared in $[17,18,24,30]$.

Consequently, by setting

$$
\bar{\xi}_{k}=\xi-\xi_{k} ; \quad \bar{\eta}_{k}=\eta-\eta_{k} \quad(23 \mathrm{a}, \mathrm{b})
$$

and by posing $\operatorname{Re}_{k}(\xi, \eta)$ and $\operatorname{Im}_{k}(\xi, \eta)$ as

$$
\begin{aligned}
& \operatorname{Re}_{k}(\xi, \eta)=\operatorname{Re}\left[f_{\zeta_{k}}(\xi, \eta)\right]=\frac{\bar{\xi}_{k}}{2} \log \left(\bar{\xi}_{k}^{2}+\bar{\eta}_{k}^{2}\right)-\bar{\eta}_{k} \arg \left(\bar{\xi}_{k}+i \bar{\eta}_{k}\right) \\
& \operatorname{Im}_{k}(\xi, \eta)=\operatorname{Im}\left[f_{\zeta_{k}}(\xi, \eta)\right]=\frac{\bar{\eta}_{k}}{2} \log \left(\bar{\xi}_{k}^{2}+\bar{\eta}_{k}^{2}\right)+\bar{\xi}_{k} \arg \left(\bar{\xi}_{k}+i \bar{\eta}_{k}\right)
\end{aligned}
$$

where $\arg (\zeta)=\arctan (\operatorname{Im}(\zeta) / \operatorname{Re}(\zeta))$ is the argument of the complex logarithm, the real and imaginary parts of $F(\zeta)$ are approximated as follows:

$$
\begin{aligned}
& \chi_{x}(\xi, \eta)=a+a_{0} \xi-b_{0} \eta+\sum_{k=1}^{n}\left[a_{k} \operatorname{Re}_{k}(\xi, \eta)-b_{k} \operatorname{Im}_{k}(\xi, \eta)\right] \\
& \chi_{y}(\xi, \eta)=b+b_{0} \xi+a_{0} \eta+\sum_{k=1}^{n}\left[b_{k} \operatorname{Re}_{k}(\xi, \eta)+a_{k} \operatorname{Im}_{k}(\xi, \eta)\right]
\end{aligned}
$$

Keeping in mind Eqs.(18a,b), accordingly the shear stress functions take the following form: 


$$
\begin{aligned}
\tau_{z x}(\xi, \eta)= & \frac{1}{R}\left(a+a_{0} \xi-b_{0} \eta+\sum_{k=1}^{n}\left[a_{k} \operatorname{Re}_{k}(\xi, \eta)-b_{k} \operatorname{Im}_{k}(\xi, \eta)\right]\right)+ \\
& +\frac{\left(m_{2}-1\right)}{R}\left(-\frac{m_{1}}{\left(m_{2}-1\right)} \frac{T_{y}}{I_{x}} \xi \eta+\frac{T_{x}}{2 I_{y}} \frac{\xi^{2}}{R}\right)-G_{z x} \bar{\theta} \eta \\
& (26 \mathrm{a}) \\
\tau_{z y}(\xi, \eta)= & -\left(b+b_{0} \xi+a_{0} \eta+\sum_{k=1}^{n}\left[b_{k} \operatorname{Re}_{k}(\xi, \eta)+a_{k} \operatorname{Im}_{k}(\xi, \eta)\right]\right)+ \\
& +\left(m_{1}-1\right)\left(-\frac{m_{2}}{\left(m_{1}-1\right)} \frac{T_{x}}{I_{y}} \frac{\xi \eta}{R}+\frac{T_{y}}{2 I_{x}} \eta^{2}\right)+R G_{z x} \bar{\theta} \xi
\end{aligned}
$$

Once the $2 n+5$ coefficients $a, a_{0}, a_{k}, b, b_{0}, b_{k}, \bar{\theta}$ are evaluated, the stress field $\boldsymbol{\tau}^{T}=\left[\begin{array}{ll}\tau_{z x} & \tau_{z y}\end{array}\right]$ in the transformed domain $\bar{A}$ is determined: in order to provide the stress distribution of the orthotropic cross-section an inverse variable transformation is required (see Eqs.11a,b).

Following the steps reported in [30] the necessary conditions for evaluating those coefficients may be obtained by expressing the free-traction boundary condition in Eq.(16) and the static equivalence conditions in Eqs.(17) in terms of the above coefficients and performing line integrals only as detailed in the following sections .

\subsection{Free-traction boundary condition on the transformed contour}

The free-traction boundary condition reported in Eq.(16) is imposed on $m$ equally spaced points of coordinate $\left(\xi_{j}, \eta_{j}\right)$ on the contour $\bar{C}$ of the transformed crosssection therefore written in the form:

$$
R \tau_{z x}\left(\xi_{j}, \eta_{j}\right) \overline{\mathrm{n}}_{\xi j}+\tau_{z y}\left(\xi_{j}, \eta_{j}\right) \overline{\mathrm{n}}_{\eta j}=0 \quad j=1, \ldots, m
$$

where $\bar{n}_{\xi j}$ and $\bar{n}_{\eta j}$ are the components of the outward normal vector to the contour $\bar{C}$ from the $j^{\text {th }}$ point. 
By substituting in Eqs.(27) the shear stresses $\tau_{z x}(\xi, \eta)$ and $\tau_{z y}(\xi, \eta)$ in terms of the unknown coefficients $a, a_{0}, a_{k}, b, b_{0}, b_{k}, \bar{\theta}$ reported in Eqs.(26), the following $m$ expressions:

$$
\begin{aligned}
& \left(a+a_{0} \xi_{j}-b_{0} \eta_{j}+\sum_{k=1}^{n}\left[a_{k} \operatorname{Re}_{k}\left(\xi_{j}, \eta_{j}\right)-b_{k} \operatorname{Im}_{k}\left(\xi_{j}, \eta_{j}\right)\right]\right) \bar{n}_{\xi j}+ \\
& \left(\left(m_{2}-1\right)\left(-\frac{m_{1}}{\left(m_{2}-1\right)} \frac{T_{y}}{I_{x}} \xi_{j} \eta_{j}+\frac{T_{x}}{2 I_{y}} \frac{\xi_{j}^{2}}{R}\right)-R G_{z x} \bar{\theta} \eta_{j}\right) \bar{n}_{\xi j}+ \\
& -\left(b+b_{0} \xi_{j}+a_{0} \eta_{j}+\sum_{k=1}^{n}\left[b_{k} \operatorname{Re}_{k}\left(\xi_{j}, \eta_{j}\right)+a_{k} \operatorname{Im}_{k}\left(\xi_{j}, \eta_{j}\right)\right]\right) \bar{n}_{\eta j}+ \\
& +\left(\left(m_{1}-1\right)\left(-\frac{m_{2}}{\left(m_{1}-1\right)} \frac{T_{x}}{I_{y}} \frac{\xi_{j} \eta_{j}}{R}+\frac{T_{y}}{2 I_{x}} \eta_{j}^{2}\right)+R G_{z x} \bar{\theta} \xi_{j}\right)=0 ; j=1, \ldots, m
\end{aligned}
$$

are provided.

It is worth to note that, for an isotropic material $\left(R=1 ; m_{1}=m_{2}=-1 /(1+v)\right)$, these conditions are the same of those provided in [30].

\subsection{Static equivalence conditions in the transformed domain}

Once the free-traction boundary condition is defined, in this section the three needed static equivalence conditions are derived.

The static equivalence conditions in $x$ and $y$-directions written in the transformed domain $\bar{A}$ and reported in Eqs.(17a,b) take the following form

$$
\begin{aligned}
& \frac{1}{R} \int_{\bar{A}} \tau_{z x} d \bar{A}=\frac{1}{R} \int_{\bar{A}}\left(\frac{\chi_{x}(\xi, \eta)}{R}+\frac{\left(m_{2}-1\right)}{R}\left(-\frac{m_{1}}{\left(m_{2}-1\right)} \frac{T_{y}}{I_{x}} \xi \eta+\frac{T_{x}}{2 I_{y}} \frac{\xi^{2}}{R}\right)-G_{z x} \bar{\theta} \eta\right) d \bar{A}=T_{x} \\
& \frac{1}{R} \int_{\bar{A}} \tau_{z y} d \bar{A}=\frac{1}{R} \int_{\bar{A}}\left(-\chi_{y}(\xi, \eta)+\left(m_{1}-1\right)\left(-\frac{m_{2}}{\left(m_{1}-1\right)} \frac{T_{x}}{I_{y}} \frac{\xi \eta}{R}+\frac{T_{y}}{2 I_{x}} \eta^{2}\right)+R G_{z x} \bar{\theta} \xi\right) d \bar{A}=T_{y}
\end{aligned}
$$

where the positions in Eqs.(18a,b) have been taken into account.

Real and imaginary parts of $F(\zeta), \chi_{x}(\xi, \eta)$ and $\chi_{y}(\xi, \eta)$,written in terms of unknown coefficients (see Eqs.25a,b) lead to: 


$$
\begin{aligned}
& \int_{\bar{A}} \chi_{x}(\xi, \eta) d \bar{A}=\int_{\bar{A}}\left(a+a_{0} \xi-b_{0} \eta\right) d \bar{A}+\sum_{k=1}^{n} \int_{\bar{A}}\left[a_{k} \operatorname{Re}(\xi, \eta)-b_{k} \operatorname{Im}_{k}(\xi, \eta)\right] d \bar{A} \\
& \int_{\bar{A}} \chi_{y}(\xi, \eta) d \bar{A}=\int_{\bar{A}}\left(b+b_{0} \xi+a_{0} \eta\right) d \bar{A}+\sum_{k=1}^{n} \int_{\bar{A}}\left[b_{k} \operatorname{Re}(\xi, \eta)+a_{k} \operatorname{Im}_{k}(\xi, \eta)\right] d \bar{A}
\end{aligned}
$$

Moreover, by recalling that $\xi$ and $\eta$ axes are the principal axes of inertia of the transformed cross section $\bar{A}$, the above static equivalence conditions, by simple calculations (see Santoro [28]) are reverted into the following form:

$$
\begin{aligned}
& \int_{\bar{A}} \chi_{x}(\xi, \eta) d \bar{A}=R^{2}\left(\frac{\left(1-m_{2}\right)}{2 R^{3}} \frac{I_{\eta}}{I_{y}}+1\right) T_{x} \\
& \int_{\bar{A}} \chi_{y}(\xi, \eta) d \bar{A}=R\left(\frac{\left(m_{1}-1\right)}{2 R} \frac{I_{\xi}}{I_{x}}-1\right) T_{y}
\end{aligned}
$$

Furthermore, the values of the first integrals on the right hand sides of Eqs.(30), are $a \bar{A}$ and $b \bar{A}$ respectively; in order to reduce the computational effort the other domain integrals have been transformed into line integrals according to Green’s lemma (see [30]).

It follows that both expressions in Eqs.(30a,b) representing the translation static equivalence conditions along the inertia axes, may be replaced with:

$$
\begin{aligned}
& a \bar{A}+\sum_{k=1}^{n}\left(\frac{a_{k}}{4} \oint_{\bar{C}} A_{k}(\xi, \eta) \bar{n}_{\eta} d \bar{C}-\frac{b_{k}}{4} \oint_{\bar{C}} A_{k}(\xi, \eta) \bar{n}_{\xi} d \bar{C}\right)=R^{2}\left(\frac{\left(1-m_{2}\right)}{2 R^{3}} \frac{I_{\eta}}{I_{y}}+1\right) T_{x} \\
& b \bar{A}+\sum_{k=1}^{n}\left(\frac{a_{k}}{4} \oint_{\bar{C}} A_{k}(\xi, \eta) \bar{n}_{\bar{\xi}} d \bar{C}+\frac{b_{k}}{4} \oint_{\bar{C}} A_{k}(\xi, \eta) \bar{n}_{\eta} d \bar{C}\right)=R\left(\frac{\left(m_{1}-1\right)}{2 R} \frac{I_{\xi}}{I_{x}}-1\right) T_{y}
\end{aligned}
$$

where

$$
A_{k}(\xi, \eta)=2 \bar{\xi}_{k} \bar{\eta}_{k}\left(\log \left(\bar{\xi}_{k}^{2}+\bar{\eta}_{k}^{2}\right)-1\right)-2\left(\bar{\eta}_{k}^{2}-\bar{\xi}_{k}^{2}\right) \arg \left(\bar{\xi}_{k}+i \bar{\eta}_{k}\right)
$$

The third static equivalence condition in Eq.(17c) after some algebraic manipulations takes the following form 


$$
\oint_{\bar{C}} \mathbf{P}^{T}(\zeta) \overline{\mathbf{s}} d \bar{C}-2 R G_{z x} \bar{\theta} I_{P}-\left(\frac{1-3 m_{2}}{R}\right) \frac{I_{\eta \eta \xi}}{I_{y}} T_{x}+\left(1-3 m_{1}\right) \frac{I_{\xi \xi \eta}}{I_{x}} T_{y}=-2 R^{2} M_{z}
$$

involving only line integrals.

Eq. (34) is written in terms of the complex potential function $F(\zeta)$ taking into account the transformation $\int_{\bar{A}} \operatorname{Im}(\zeta F(\zeta)) d \bar{A}=\frac{1}{2} \oint_{\bar{C}} \mathbf{P}^{T}(\zeta) \mathbf{s} d \bar{C}$ as detailed in [32] where the formal vector $\mathbf{P}^{T}=\left[\begin{array}{ll}p & q\end{array}\right]$ is composed by real and imaginary parts of the function $P(\zeta)=p+i q$ that is the primitive function of $\zeta F(\zeta)$; $\overline{\mathbf{s}}^{T}=\left[\begin{array}{ll}\bar{S}_{x} & \bar{S}_{y}\end{array}\right]$ is the unit vector tangent to the contour $\bar{C}$ of the cross-section. Moreover, in Eq. (34) $I_{p}=\int_{\bar{A}}\left(\xi^{2}+\eta^{2}\right) d \bar{A}$ is the polar inertia moment and $I_{\xi \eta \eta}=\int_{\bar{A}} \xi^{2} \eta d \bar{A}$ and $I_{\eta \xi \xi}=\int_{\bar{A}} \eta^{2} \xi d \bar{A}$ are the third order inertia moments, respectively: all these quantities, following the philosophy of the CVBEM, are transformed into line integrals (see [21-23, 27-30, 32]) and evaluated in the transformed boundary $\bar{C}$.

By taking into account the expression of $F(\zeta)$ in Eqs.(21), the corresponding primitive function $P(\zeta)$ of $\zeta F(\zeta)$ is written in terms of unknown coefficients as

$$
\begin{aligned}
& P(\zeta)=\frac{1}{6}\left(3 \alpha \zeta^{2}+2 \alpha_{0} \zeta^{3}\right)+ \\
& +\frac{1}{36} \sum_{k=1}^{n} \alpha_{k}\left(-4 \zeta^{3}+3 \zeta^{2} \zeta_{k}+6 \zeta \zeta_{k}^{2}-27 \zeta_{k}^{3}+6\left(\zeta-\zeta_{k}\right)^{2}\left(2 \zeta+\zeta_{k}\right) \log _{\hat{\zeta}_{k}}\left(\zeta-\zeta_{k}\right)\right)
\end{aligned}
$$

The latter expression, as a formal vector $\mathbf{P}^{T}=\left[\begin{array}{ll}p & q\end{array}\right]$, is given considering the real part $p(\xi, \eta)$ and the imaginary one $q(\xi, \eta)$ in terms of the coefficients $a, a_{0}, a_{k}, b, b_{0}, b_{k}, \bar{\theta}$ as follows: 


$$
\begin{aligned}
\begin{aligned}
& p(\xi, \eta)= \frac{1}{6}\left((a u-b v)+\left(a_{0} u_{0}-b_{0} v_{0}\right)\right)+ \\
&+\frac{1}{36} \sum_{k=1}^{n}[\left(a_{k} u_{k}-b_{k} v_{k}\right)+\left(a_{k} \hat{u}_{k}-b_{k} \hat{v}_{k}\right) \log \left(\left(\xi-\xi_{k}\right)^{2}+\left(\eta-\eta_{k}\right)^{2}\right)+ \\
&\left.\quad-2\left(a_{k} \hat{v}_{k}+b_{k} \hat{u}_{k}\right) \arg \left(\left(\xi-\xi_{k}\right)+i\left(\eta-\eta_{k}\right)\right)\right] \\
& q(\xi, \eta)= \frac{1}{6}\left((a v+b u)+\left(a_{0} v_{0}+b_{0} u_{0}\right)\right)+ \\
&+\frac{1}{36} \sum_{k=1}^{n}\left[\left(a_{k} v_{k}+b_{k} u_{k}\right)+\left(a_{k} \hat{v}_{k}+b_{k} \hat{u}_{k}\right) \log \left(\left(\xi-\xi_{k}\right)^{2}+\left(\eta-\eta_{k}\right)^{2}\right)+\right. \\
&\left.\quad+2\left(a_{k} \hat{u}_{k}-b_{k} \hat{v}_{k}\right) \arg \left(\left(\xi-\xi_{k}\right)+i\left(\eta-\eta_{k}\right)\right)\right]
\end{aligned}
\end{aligned}
$$

where the positions reported in appendix have been taken into account [30].

\section{Numerical applications}

In this last section, the proposed method is implemented to compute the shear stresses for three orthotropic cross-sections subjected to external shear and twist moments. An elliptic cross-section is first used as benchmark test, since for this shape the exact solution is known in closed form. Then, solutions for a rectangle and a triangular equilateral cross-section are compared with results proposed in literature.

The shear stress functions, written in terms of the complex potential expanded through the CVBEM series as in Eq. (26), can be determined once the $2 n+4$ series coefficients and the unitary twist rotation of the cross-section are calculated. The first step in the implementation is to operate the variable transformation Eq. (11) and select $n$ nodes and $m$ collocation points on the contour $\bar{C}$ of the transformed domain. Then, the unknown coefficients are obtained as solution of the set of algebraic equations composed of the three static equivalence conditions with respect to translations and rotation of the cross-section, Eqs. (31) and Eq. (34), respectively, and of the free-traction boundary condition Eq. (28) expressed in correspondence of the $m$ collocation points. In particular, for the following numerical applications, all results have been obtained using $m=3 n+5$ and the resultant over-determined system of algebraic equations has been solved by the pseudo-inverse matrix method, obtaining, therefore, the best solution in the least square sense. In agreement with the formulation proposed in the previous sections, the shear forces act along the principal inertia axes. The geometrical 
parameters of the studied domains, as well as the values of the external forces, have been selected to compare the obtained results with those presented in [28]. The first domain is the orthotropic elliptical cross-section depicted in Figure 3a, having semiaxes $a=2$ and $b=1$. It has been considered $R=\frac{\sqrt{2}}{2}, m_{1}=0.06$ and $m_{2}=0.02$. Two different cases have been analysed. In the first one, an external unitary twist moment $\left(M_{z}=1\right)$ is applied to the beam. For this case, the closed form of the shear stress functions is the same of the isotropic case [4]:

$$
\begin{aligned}
& \tau_{z x}(x, y)=-\frac{2 y}{\pi a b^{3}} \\
& \tau_{z y}(x, y)=\frac{2 x}{\pi a^{3} b}
\end{aligned}
$$

while the exact value of the unitary twist rotation of the cross-section can be calculated as:

$$
\theta=\frac{a^{2} R^{2}+b^{2}}{\pi a^{3} b^{3} R^{2} G_{z x}}
$$

The shear stress field modulus, obtained by CVBEM is depicted in Figure 4a, while the resulting value of the unitary twist rotation of the cross-section is $\theta=0.026873$, in perfect agreement with the exact solution. Figure $4 \mathrm{~b}$ shows the shear stress functions $\tau_{z x}$ and $\tau_{z y}$ evaluated along a line parallel to the $x$ axis with $y=0.25$, comparing exact solution and numerical results by CVBEM. Analogous results are reported for the case of an external shear force acting along the $y$ axis $\left(T_{y}=1\right)$. In this case the analytic expression for the shear stresses is [4, 28]:

$$
\begin{aligned}
& \tau_{z x}(x, y)=-\frac{4\left(b^{2}+a^{2} R^{2} m_{1}\right)}{\pi a b^{3}\left(3 b^{2}+a^{2} R^{2}\right)} x y T_{y} \\
& \tau_{z y}(x, y)=\frac{2\left(\left(2 b^{2}+a^{2} R^{2}\left(1-m_{1}\right)\right)\left(b^{2}-y^{2}\right)+b^{2} R^{2}\left(3 m_{1}-1\right) x^{2}\right)}{\pi a b^{3}\left(3 b^{2}+a^{2} R^{2}\right)} T_{y}
\end{aligned}
$$

The shear stress modulus inside the domain, obtained by CVBEM, and the shear stress along a horizontal line at $y=0.25$, both for the numerical and the analytical cases, are depicted in Figures 5a and 5b, respectively. For the pure torsion and the pure shear cases, $n=12$ uniformly distributed nodes have been selected on the contour of the transformed domain. 
The second analysed domain is a rectangle having sides $a$ and $b$ (Figure 3b). For this domain as well, two different load cases have been considered, so that results could be compared with those proposed in [27] and [28]. In the first case, a unitary external twist moment is considered $\left(M_{z}=1\right)$. Since this is a pure torsion case, only the $R$ parameter is relevant, since $m_{1}$ and $m_{2}$ affect the results exclusively if the external shear forces are not null. The shear stress modulus for $a=4, b=3$ and $R=3$, using CVBEM with $n=30$ nodes on the transformed contour, is plotted in Figure 6a and results are in perfect agreement with [27]. The value $\theta=0.03295$ is obtained for the unitary twist rotation of the cross-section, accordingly with the value proposed in [4]:

$$
\theta=\frac{M_{z}}{0.281 G_{z x} a b^{3}}=0.03295
$$

A second rectangular domain has been studied for the pure shear case $\left(T_{y}=1\right)$, selecting side lengths $a=4$ and $b=2$ and parameters $R=\sqrt{2} / 2, m_{1}=0.06$ and $m_{2}=0.02$, (reproducing the solutions proposed in [28]), using CVBEM with $n=30$ nodes. Results are shown in Figure $6 \mathrm{~b}$ in terms of shear stress modulus. For the elliptical and rectangular domains, the convergence of the method is very fast, and, as shown, few nodes are needed.

Finally, an equilateral triangular cross-section with unitary length side has been studied. Also for this domain the two cases of external torsion and external shear have been considered ( $M_{z}=1$ and $T_{x}=1$ ), considering $R=0.5$ and, for the shear case, $m_{1}=0.12$ and $m_{2}=0.02$. It is worth to stress that, for the case of $T_{x} \neq 0$, since the shear centre does not coincide with the origin of the reference axes, an induced rotation of the cross-section has to be expected. Numerical results obtained by CVBEM for increasing number of nodes on the transformed domain have been compared with results obtained by LEM varying the order of truncation of the Laurent series [28], and they are reported in Table 1 in terms of values of $\theta$ for both torsion and shear. It is worth to note that, for both cases of torsion and shear actions. For both cases, the two methods converge towards the same value. While the CVBEM implementation provide an underestimation of the values of $\theta$, the results obtained by LEM are, instead, an overestimation of the correct value. Analogously to the other domains, the shear stress modulus for the two 
studied cases, obtained for $n=270$ nodes, are shown in Figure 7a and 7b, respectively.

\section{Conclusions}

In this paper a modified Complex Variable Boundary Element Method is employed to provide a solution for the coupled flexure-torsion De Saint Venant problem for orthotropic beams. The analysis, combined with a suitable transformation of coordinates, is formulated by employing a complex potential function whose real and imaginary parts are related to the shear stress components, the orthotropic ratio and the Poisson coefficients.

By applying the proposed method, the complete shear stress distribution and the unitary twist rotation of the cross-section at once can be achieved by performing only line integrals, respecting the philosophy of the boundary methods.

Numerical applications for the solution of De Saint Venant torsion and flexure problem, for different cross-section geometries, are reported to show the validity and the efficiency of the proposed method in presence of orthotropic material. Results obtained for an elliptical cross-section under torsion and shear excitations have been considered as a benchmark solution to verify the accuracy of the method. Other cross-section geometries have been studied to investigate its stability and the convergence of the applied series for increasing number of nodes in the boundary discretization.

\section{Appendix}

In section 4.2, the function $P(\zeta)=p+i q$ has been introduced to formulate the static equivalence condition with respect to rotations of the cross-section on its plane involving only line integrals. The function $P(\zeta)$ is obtained as the primitive of the function $\zeta F(\zeta)$, where $F(\zeta)$ is defined in Eq. (21). Real and imaginary parts of the function $P(\zeta)$ can be expressed in terms of the series 
coefficients $a, a_{0}, \ldots, a_{k}, b, b_{0}, \ldots, b_{k}$ and of the unitary twist rotation of the crosssection as in Eq. (36), where the following positions have been made:

$$
\begin{gathered}
u(\xi, \eta)=3\left(\xi^{2}-\eta^{2}\right) \\
v(\xi, \eta)=6 \xi \eta \\
u_{0}(\xi, \eta)=2 \xi\left(\xi^{2}-3 \eta^{2}\right) \\
v_{0}(\xi, \eta)=-u_{0}(\eta, \xi) \\
u_{k}(\xi, \eta)=-4 \xi^{3}+3 \xi^{2} \xi_{k}-27 \xi_{k}^{3}-3 \xi_{k} y^{2}-12 \xi_{k} \eta \eta_{k}+81 \xi_{k} \eta_{k}^{2}+\xi\left(6 \xi_{k}^{2}+12 \eta^{2}-6 \eta \eta_{k}+6 \eta_{k}^{2}\right) \\
v_{k}(\xi, \eta)=-u_{k}(\eta, \xi)
\end{gathered}
$$

$$
\begin{aligned}
& \hat{u}_{k}(\xi, \eta)=6 \xi^{3}-9 \xi^{2} \xi_{k}+3 \xi_{k}^{3}-18 \xi \eta^{2}+9 \xi_{k} \eta^{2}+18 \xi \eta \eta_{k}-9 \xi_{k} \eta_{k}^{2} \\
& \hat{v}_{k}(\xi, \eta)=-\hat{u}_{k}(\eta, \xi)
\end{aligned}
$$

\section{References}

[1] Love A.E.H.: A Treatise on the Mathematical Theory of Elasticity. Fourth edition Dover Publications, New York (1926)

[2] Sokolnikoff I.S.: Mathematical theory of elasticity. McGraw-Hill, New York (1956)

[3] Timoshenko S.P., Goodier J.N.: Theory of elasticity. McGraw-Hill, New York (1970)

[4] Lekhnitskii S.G.: Theory of Elasticity of an Anisotropic Body. Mir Publishers, Moscow (1981)

[5] Tolf G.: St. Venant bending of an orthotropic beam. Composite Structures. 4, 1-14 (1985)

[6] Kosmatka J.B., Dong S.B.: Saint-Venant solutions for prismatic anisotropic beams. International Journal of Solids and Structures. 28 (7), 917-938 (1991)

[7] Kosmatka J.B.:Flexure-torsion Behavior of Prismatic Beams, Part I: Section Properties via Power Series. AIAA Journal. 31(1), 170-179 (1993)

[8] Chou S.I.: Determination of centers of flexure using the boundary element method.

Engineering Analysis with Boundary Elements. 12, 321-324(1993).

[9] Mokos V.G., Sapountzakis E.J.: A BEM solution to transverse shear loading of beams. Comput Mech. 36, 384-97(2005a)

[10] Sapountzakis E.J., Mokos V.G.: A BEM solution to transverse shear loading of composite beams. International Journal of Solids and Structures. 42, 3261-3287(2005b)

[11] Sapountzakis E.J., Protonotariou V.M.: A displacement solution for transverse shear loading of beams using the boundary element method. Computers and Structures. 86 (7-8), 771-779(2008) 
[12] Gaspari D., Aristodemo M.: Torsion and flexure analysis of orthotropic beams by a boundary element model. Engineering Analysis with Boundary Elements. 29 850-858(2005).

[13] Lacarbonara W., Paolone A.:,On solution strategies to Saint-Venant problem. Journal of Computational and Applied Mathematics. 206 (1), 473-497(2007).

[14] Muskhelishvili N.I.: Some Basic Problems of the Mathematical Theory of Elasticity.

P.Noordhoff Ltd., Groningen-The Netherlands (1953).

[15] England AH.: Complex variable methods in elasticity. Wiley-Interscience (1971).

[16] Hromadka II TV.: The complex variable boundary element method. Berlin, Heidelberg, New York, Tokyo, Springer-Verlag (1984)

[17] Hromadka II TV, Whitley RJ.: Advances in the complex variable boundary element method.London Limited, Springer-Verlag (1998)

[18] Whitley RJ, Hromadka II TV.: Theoretical developments in the complex variable element method. Engineering Analysis with Boundary Elements. 30, 1020-1024(2006)

[19] Hromadka II TV, Guymon LG.: Complex polynomial approximation of the Laplace equation. Journal of Hydraulic Engineering. 110(3), 329-39(1984)

[20] Poler AC, Bohannon AW, Schowalter SJ, Hromadka II TV.: Using the complex polynomial method with mathematica to model problems involving the Laplace and Poisson equations Published online in Wiley Interscience (www.interscience.wiley.com) (2008) DOI 10.1002/num.20365

[21] Di Paola M., Pirrotta A., Santoro R., Line Element-less Method (LEM) for beam torsion solution (truly no-mesh method). Acta Mechanica. 195, 349-63(2008)

[22] Di Paola M., Pirrotta A., Santoro R.: De Saint-Venant flexure-torsion problem handled by Line Element-less Method (LEM). Acta Mechanica. 217, 101-118(2011)

[23] Pirrotta A., LEM for twisted re-entrant angle sections. Computer and Structures. 133, 149-155 (2014)

[24] Barone G., Pirrotta A., Santoro R.: Comparison among three boundary element methods for torsion problems: CPM, CVBEM, LEM. Engineering Analysis with Boundary Elements. 35, 895907(2011)

[25] Aleynikov S.M., Stromov A.V.,: Comparison of complex methods for numerical solutions of boundary problems of the Laplace equation. Engineering Analysis with Boundary Elements. 28, 615-622 (2004).

[26] Dumir P.C., Kumar R.: Complex variable boundary element method for torsion of anisotropic Bars. Applied Mathematical Modelling. 17, 80-88 (1993)

[27] Santoro R.: The line element-less method analysis of orthotropic beam for the De Saint Venant torsion problem. International Journal of Mechanical Sciences. 52, 43-55(2010)

[28] Santoro R.: Solution of De Saint Venant flexure-torsion problem for orthotropic beam via LEM (Line Element-less Method). European Journal of Mechanics A/Solids. 30, 924-939(2011)

[29] Barone G., Pirrotta A.: CVBEM application to a novel potential function providing stress field and twist rotation at once. Journal of Engineering Mechanics. 9 (139), 1290-1293(2013)

[30] Barone G., Pirrotta A.: CVBEM for solving De Saint-Venant solid under shear forces.

Engineering Analysis with Boundary Elements. 37 (2) 197-204 (2013) 
[31] Barone G., Lo Iacono F., Navarra G.: Complex potential by hydrodynamic analogy for the determination of flexure-torsion induced stresses in de Saint Venant beams with boundary singularities. Engineering Analysis with Boundary Elements. 37 (12), 1632-1641(2013)

[32] Pirrotta A.: Complex potential function in elasticity theory: Shear and torsion solution through line integrals. Acta Mechanica. 223 (6) 1251-1259 (2012)

\section{Figure Captions}

Fig.1. De Saint Venant cylinder under coupled bending and torsion.

Fig.2. Domain transformation.

Fig.3a. Elliptical cross-section.

Fig.3b. Rectangular cross-section.

Fig.3c. Triangular cross-section.

Fig.4. Elliptical cross-section under twist moment $\left(M_{z}=1, R=\sqrt{2} / 2\right)$ : a) shear stress field modulus via CVBEM; b) exact (continuous lines) and CVBEM (dashed lines) solutions for shear stress functions $\tau_{z x}$ and $\tau_{z y}$ along x-axis.

Fig.5. Elliptical cross-section under shear force $\left(T_{y}=1, R=\sqrt{2} / 2, m_{1}=0.06, m_{2}=0.02\right)$ : a) shear stress field modulus via CVBEM; b) exact (continuous lines) and CVBEM (dashed lines) solutions for shear stress functions $\tau_{z x}$ and $\tau_{z y}$ along x-axis.

Fig.6. Rectangular cross-section: shear stress field modulus due to: a) twist moment $\left(M_{z}=1, R=1 / 2\right)$ and $\left.\mathrm{b}\right)$ shear force $\left(T_{y}=1, R=\sqrt{2} / 2, m_{1}=0.06, m_{2}=0.02\right)$.

Fig.7. Triangular cross-section: shear stress field modulus due to: a) twist moment $\left(M_{z}=1, R=1 / 2\right)$ and $\left.\mathrm{b}\right)$ shear force $\left(T_{x}=1, R=\sqrt{2} / 2, m_{1}=0.06, m_{2}=0.02\right)$.

\section{Table Caption}

Table 1. Unitary twist rotation of the equilateral triangular cross-section for the cases of $M_{z}=1$ and $T_{x}=1$ by CVBEM and LEM. 
Figure

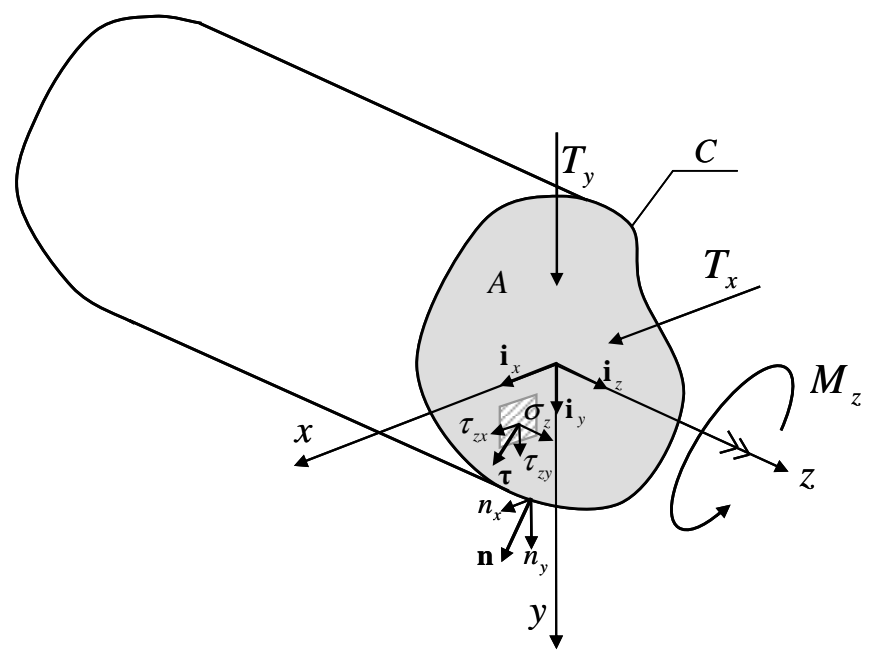

FIGURA 1 

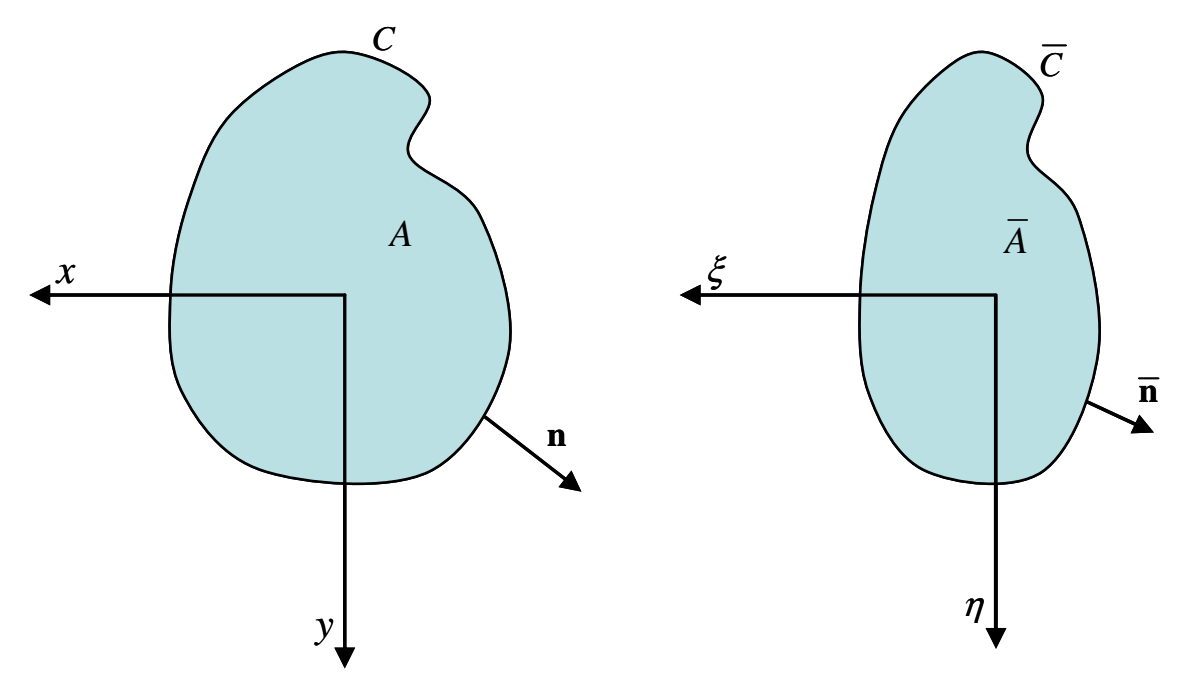

FIGURE 2

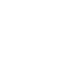

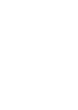

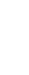

Figure

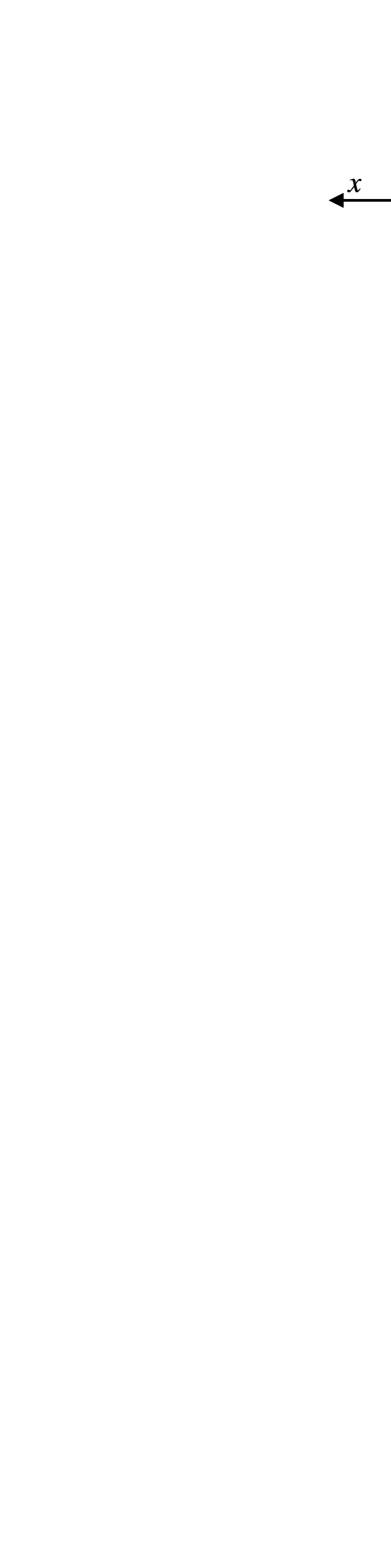




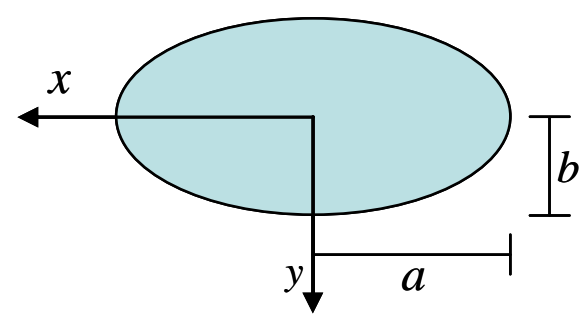

(a)

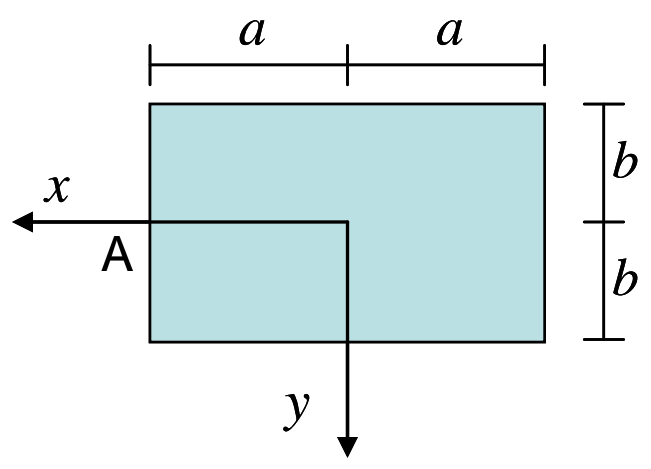

(b)

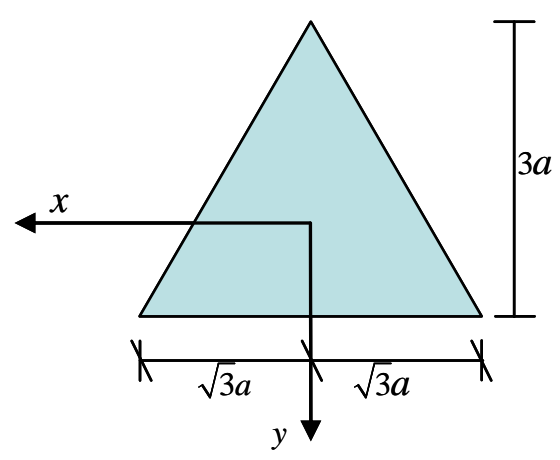

(c)

FIGURE 3 
a)

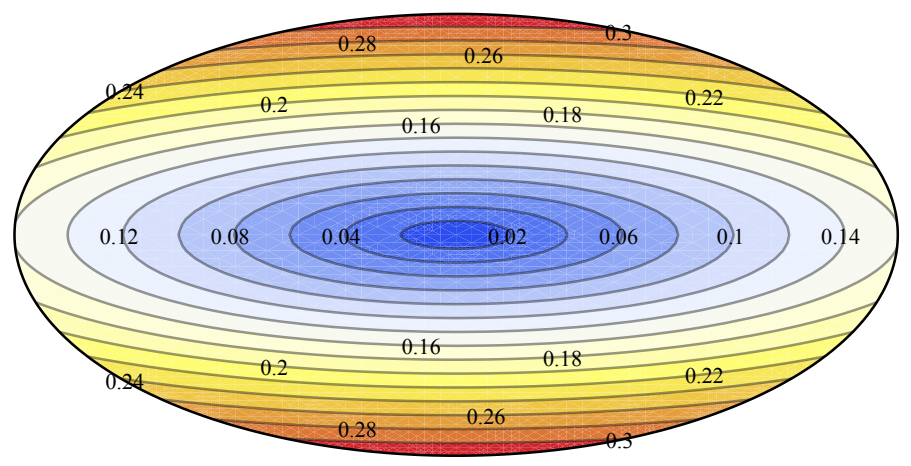

b) - $\tau_{z x}$ Exact

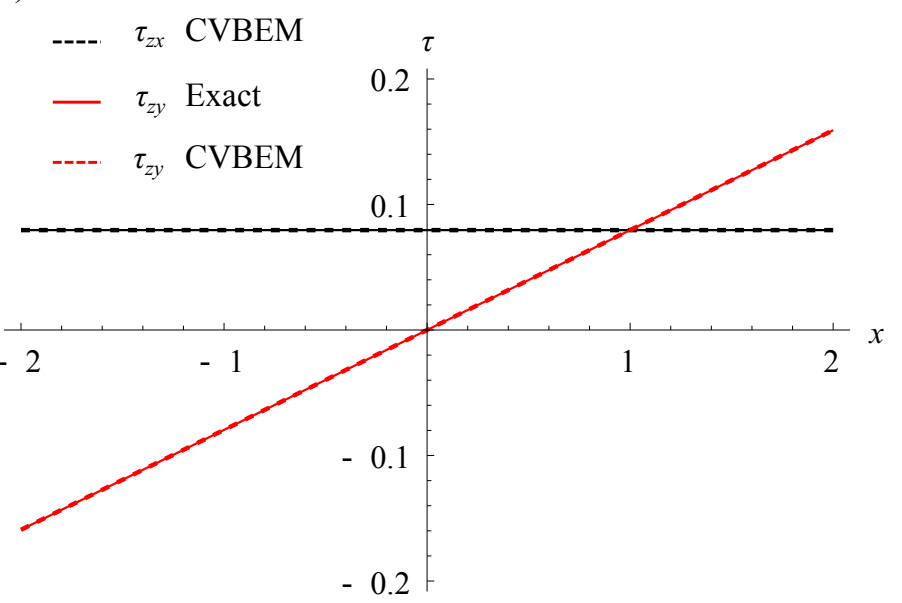


a)

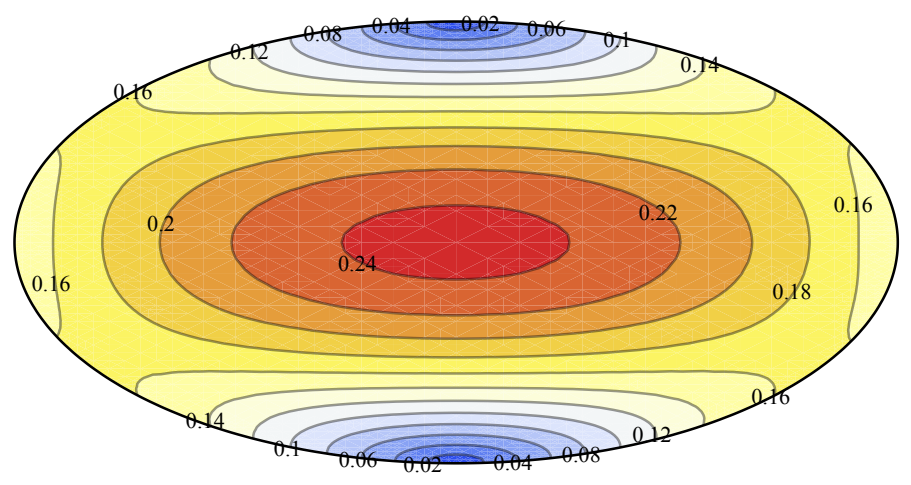

b) - $\tau_{z x}$ Exact

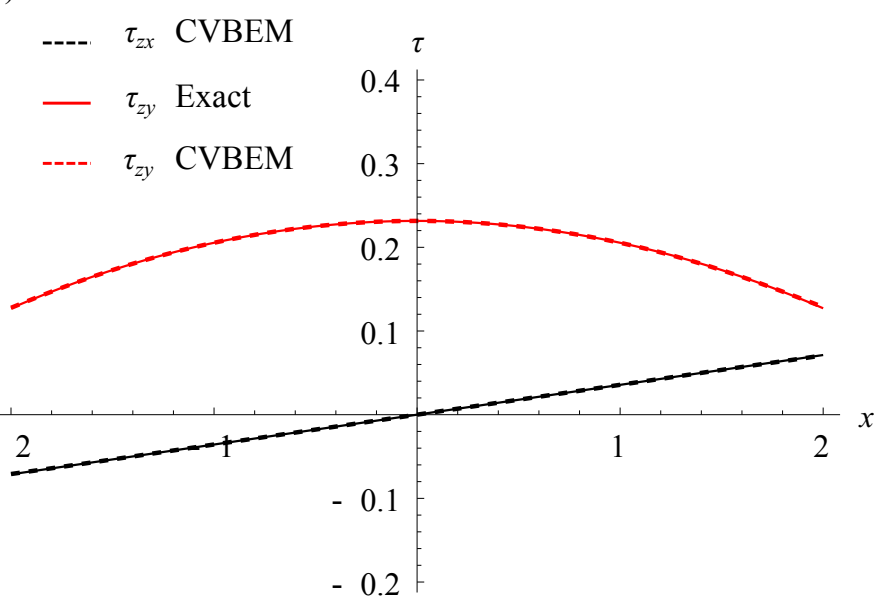


a)

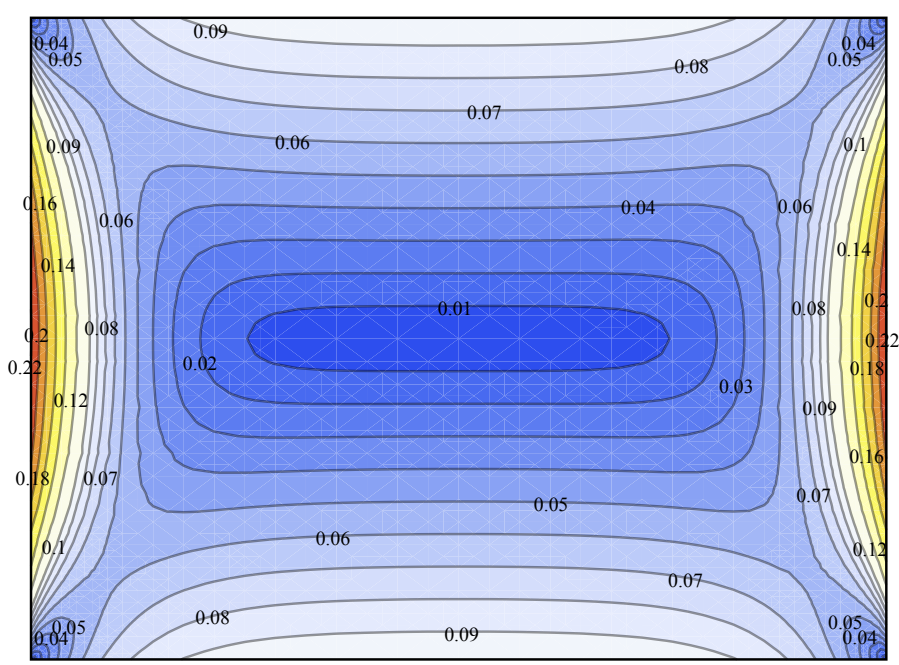

b)

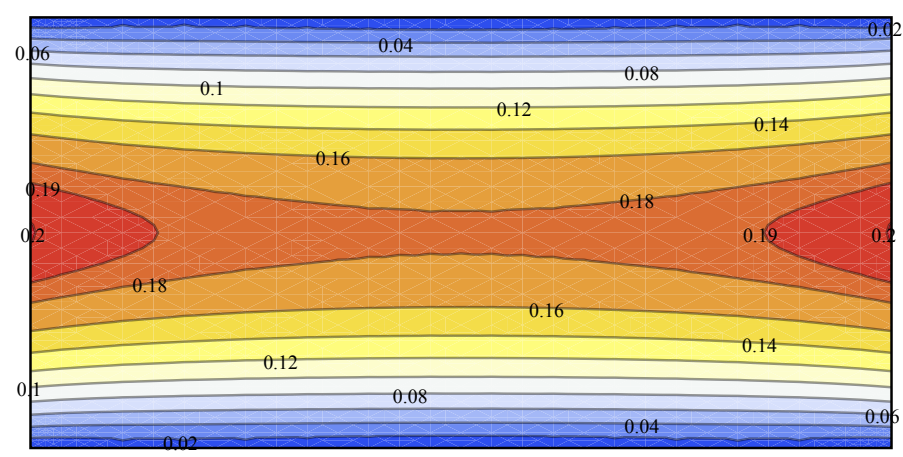

FIGURE 6 
a)

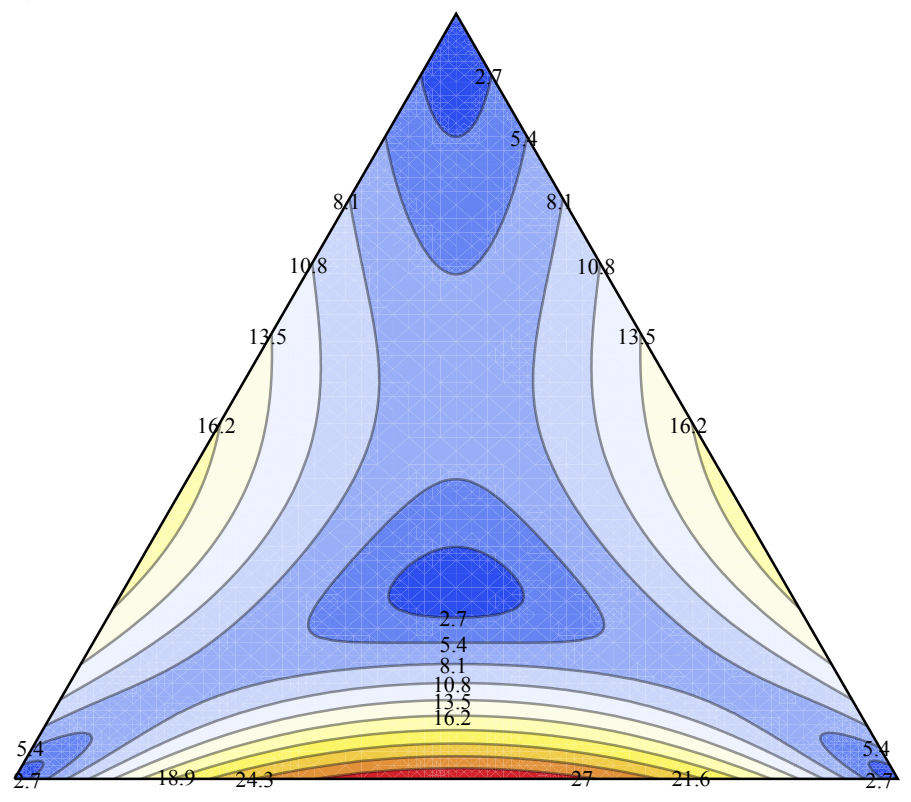

b)

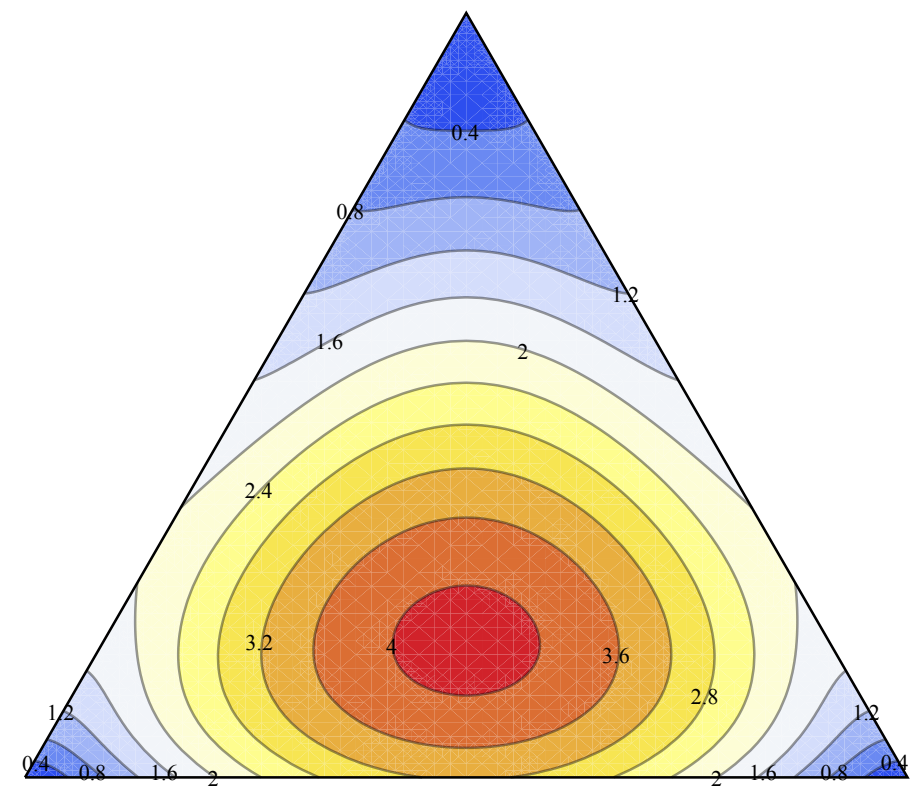

FIGURE 7 


\begin{tabular}{ccc|ccc}
\hline \multicolumn{3}{c|}{ CVBEM } & \multicolumn{4}{c}{ LEM } \\
\hline Nodes & $\theta$ for $M_{z}=1$ & $\theta$ for $T_{x}=1$ & Truncation & $\theta$ for $M_{z}=1$ & $\theta$ for $T_{x}=1$ \\
\hline 90 & 5.32878 & 0.336062 & 5 & 5.37163 & 0.341950 \\
180 & 5.34626 & 0.338235 & 6 & 5.35832 & 0.339949 \\
270 & 5.34887 & 0.338569 & 7 & 5.35197 & 0.339002 \\
360 & 5.34961 & 0.338662 & 8 & 5.35123 & 0.338892 \\
450 & 5.34991 & 0.338702 & 9 & 5.35119 & 0.338886 \\
540 & 5.35008 & 0.338724 & 10 & 5.35075 & 0.338819 \\
\hline
\end{tabular}

Table 1 